Odile Calame

BIH/CERGA

Ave. Copernic

06130 Grasse, France

\title{
INTRODUCTION
}

The status of the EROLD campaign has already been described in another paper (Mulholland, 1979). Since the beginning of 1977 all of the computer software has been ready to work. With the expectation that at least one other station would be able to operate before the end of 1977 , it appeared not very interesting to use the single-station data for the study of UTO during the last year since the other parameters of the Earth's rotation can be determined only with multiple stations. However, as this situation of a single operating station continues, it seemed to be preferable to attempt some determinations of UT0, even if that represents only a part of the goals envisioned from this campaign. We give here a brief resume of our preliminary results.

\section{DETERMINATION OF UTO}

The determinations of UTO have been made for the available data from the McDonald station, using four lunar reflectors. Except for some improvements, the mathematical model used to compute the residuals and partial derivatives has already been described elsewhere (Calame, 1976); recently the effects of tides for an elastic Earth model have been introduced.

Several determinations have been made for UTO differing by the selection of the data and the time intervals for which each value of UTO was determined. For example, averaging has been performed over 5 days, 2 days, and 1 day with the constraints that the extreme hour angles in each interval differ by at least 3 hours and that the number of data be greater than 5 to 10. A first fitting pass consisted of an adjustment for 46 parameters describing the motions of the reflectors with respect to the center of mass of the Earth. In addition, some unknowns for the geocentric position of the station were included: coordinates, drift in longitude, annual term in longitude, and a long-period term (18.6 years). In a second step, new post-fit residuals were computed and another adjustment was made to take into consideration the geocentric unknowns 
for the station (mentioned above) and corrections to UT0 taken from the 5-day smoothed values published by the $\mathrm{BIH}$ and used in the residual computations.

Figure 1 shows a sample of the results obtained for UT0 from 2-day determinations for the year 1977. For the moment, it appears to be difficult to detect any short-period effects with confidence. The rms residual in the time interval 1970 - 1978 is about 36 centimeters.

\section{JUTO (meDonake)}

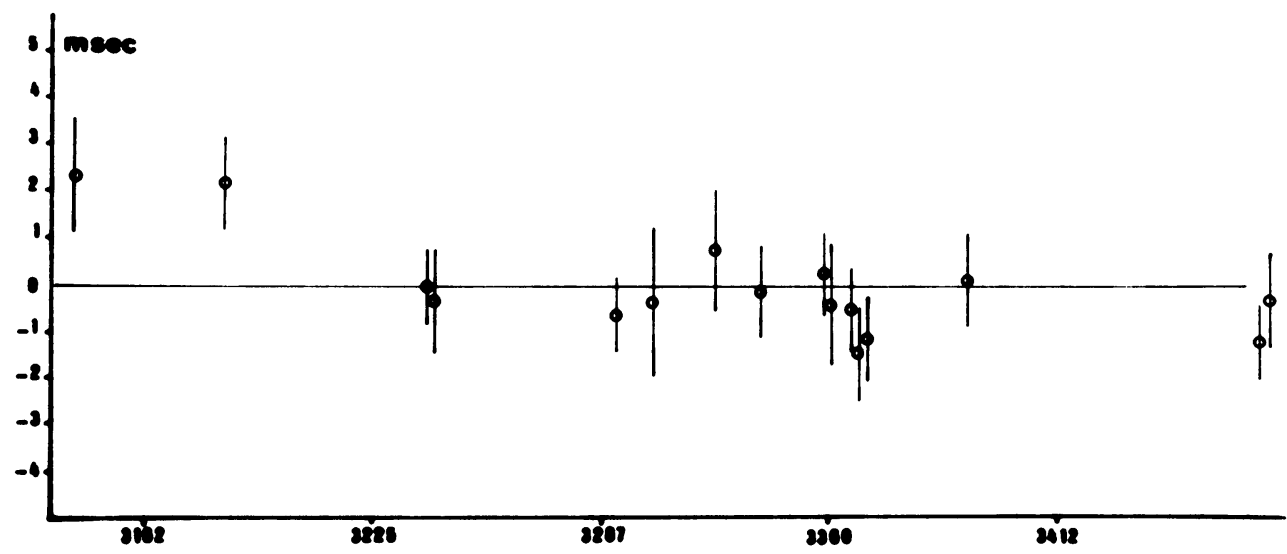

jeers Jultens - 200ens.

Figure 1. Corrections to BIH UTO determined from EROLD data.

\section{CONCLUSION}

It is necessary to emphasize that these results concern only UT0 determinations. It would be unrealistic to compare them with UT1 determinations obtained in other ways. Indeed, some local effects may perturb the results for a single station so that the global Earth rotation may differ from them. Only when other stations become operational will it be possible to study this question along with polar wander. As soon as data from other stations will be available we will be able to get new results very quickly, for example, in one or two weeks.

\section{REFERENCES}

Calame, 0.: 1976, Manuscripta Geodaetica, vol. 1, no. 3. Mulholland, J. D.: 1979, this volume. 